\title{
Sociocultural Context and the DSM-5 Alternative Model of Personality Disorder: Comment on Widiger \& Hines
}

\author{
Craig Rodriguez-Seijas, Ph.D. \\ Department of Psychology \\ University of Michigan
}

This manuscript has been accepted for the journal Personality Disorders: Theory, Research, and Treatment. It has not been copy edited. It is not the copy of record.

Correspondence concerning this article can be addressed to Craig Rodriguez-Seijas, Department of Psychology, University of Michigan, 2215 East Hall, 530 Church Street, Ann Arbor, MI 48109-1043. E-mail: crseijas@umich.edu 


\begin{abstract}
Widiger and Hines provide a brief overview of the development of the Alternative Model of Personality Disorder (AMPD) housed within Section 3 of the fifth edition of the Diagnostic and Statistical Manual of Mental Disorders (DSM-5; American Psychiatric Association, 2013). They highlight eight issues and controversies related to the AMPD in need of resolution for improvement of both the AMPD model itself as well as the field of personality disorders more broadly. In this brief commentary, I add a ninth issue in need of attention both with respect to the AMPD but also within the field of personality disorders more broadly: 9) How is sociocultural context to be accommodated in AMPD—and more generally personality disorder_theory, research, and treatment? The historical intra-individual, deficit-based models for conceptualizing personality disorders linger in current personality disorder discourse. However, failure to appropriately consider sociocultural context that systematically predisposes wide swaths of the population to unequal access to resources and exposure to psychological stressors, which can impact the appearance of personality pathology, serves to stigmatize minoritized individuals. The personality disorder field, and the AMPD discourse, must appropriately contend with sociocultural context in its models otherwise it risks developing models with limited generalizability and which hold potential to adversely affect sexual and gender minoritized populations, among others.
\end{abstract}

Keywords: Alternative Model of Personality Disorder; Sexual and Gender Minority; LGBTQ+; Sociocultural Context; Personality Disorders 
Sociocultural Context and the DSM-5 Alternative Model of Personality Disorder: Comment on Widiger and Hines

Widiger and Hines highlight eight issues that must be tackled in current and future research on the Alternative Model of Personality Disorder (AMPD): (1) Does the DSM-5 AMPD have any incremental validity or utility over DSM-IV? (2) What are the treatment implications for the AMPD? (3) Is the Level of Personality Functioning (LPF) a unitary construct? (4) Does the LPF define the core of personality disorder? (5) Is the LPF even necessary? (6) Is the DSM-5 trait model sufficiently comprehensive in its coverage? (7) Synchronization of the ICD-11 and DSM-5. (8) Should any DSM-IV syndrome be retained? By their own admission, this list of questions is not exhaustive. There are likely myriad other issues with which personality — and AMPD — researchers and stakeholders must contend.

The purpose of this commentary is to add and briefly discuss one additional question to this list: (9) How is sociocultural context to be accommodated in AMPD—and personality disorder more broadly — theory, research, and treatment? Contending with the sociocultural context necessarily complicates both the AMPD as well as the field of personality pathology. For example, when it comes to questions 1, 3, 4, and 5-pertaining to the definition of personality disorders - sociocultural context begets questions about how structural determinants of health have been included in historical and contemporary personality disorder models. Question 2, about treatment implications, raises questions of how personality disorder interventions depart in subtle, yet arguably fundamental, ways from evidence-based intervention principles for working with historically excluded populations (harking back to issues of personality disorder epistemology). Question 6, regarding 
comprehensiveness of Criterion B traits, highlights issues of inclusion; to what extent have diverse populations been included in the creation and examination of assessment measures?

It is my assertion that the field of personality disorder theory has largely operated in a manner that is socially decontextualized from the broader structural environment in which individuals exist. The assumptions that persist within the contemporary discourse on the AMPD continue to reflect some of the intra-individual, deficit-based models of earlier personality disorder theories. The net effect is ignorance of the ways in which systematic oppression creates chronically stressful environments that do not equally apply to or affect all members of the population. By failing to situate personality pathology appropriately in the sociocultural context of the individual and ignoring systematic ways in which certain populations are differentially impacted by these social structures, the field of personality disorder inadvertently propagates stigma of its own. Accommodating sociocultural context within personality disorder and AMPD discourse is overdue.

In terms of structure, I first discuss my positionality relative to the personality discourse. Next, I briefly highlight the historical deficiency-based roots of personality disorder theory. I then illustrate how neglect of sociocultural context impacts conceptualization of personality pathology as well as its treatment, corresponding with questions 4 and 2 respectively, as raised by Widiger and Hines. I end by calling for increased attention to context in the study of personality pathology both in relation to the AMPD and more broadly.

\section{Author Positionality Statement}

On a professional level, I study transdiagnostic dimensional models of psychopathology — like the AMPD — and factors associated with manifestation of psychopathology among minoritized groups. My research has included investigating how 
minority stressors relate transdiagnostically to psychopathology outcomes (e.g., RodriguezSeijas, Eaton, et al., 2019; Rodriguez-Seijas et al., 2015). My clinical training lies in the provision of evidence-based, affirming care to sexual and gender (SGM) populations (e.g., Rodriguez-Seijas, Burton, et al., 2019) and in training graduate students in conceptualizing psychopathology with an eye to sociocultural context. Most recently, my research has broadened into the personality disorder realm, focusing on issues related to assessment and diagnosis of borderline personality disorder among SGM populations (e.g., Rodriguez-Seijas et al., 2021a, 2021b). On a personal level, I possess several minoritized identities including sexual and racial/ethnic minority, and immigrant statuses. These professional and personal experiences form the backdrop for this commentary, and explain my reference to SGM populations throughout, though the principles I outline herein arguably apply to other understudied and excluded populations.

\section{Deficit-Based Conceptualization of Personality Disorder}

The lineage of personality disorder theory is one of constitutional deficiency. Crocq (2013) reviewed major theories of personality disorders/personality pathology which largely reflect intra-individual deficit models. For instance, Kraepelin considered psychopathic personalities the result of inborn defect. Similarly, Schneider theorized that abnormal personalities resulted from largely inborn, constitutional factors. This intra-individual deficiency narrative permeates earlier instantiations of the DSM. In DSM-I, personality disorders are "characterized by developmental defects or pathological trends in personality structure" wherein personality is “...considered deep seated disturbances, with little room for regression” (p. 34). In DSM-II, personality pathology results from "deeply ingrained maladaptive patterns of behavior" (p. 41) dropping the explicit reference to inborn defect. 
From DSM-III and beyond, personality disorders are described as enduring patterns of perceiving and responding to the world in a maladaptive manner.

Sections 2 and 3 of the DSM-5 explicitly consider how an individual's cultural expectations impact the diagnosis of a personality disorder. Within Section 2, personality disorders "deviate markedly from the expectations of an individual's culture" (p. 629) and are "... distinguished from characteristics that emerge in response to specific situational

stressors ..." (p. 630). Within the AMPD, caution is suggested about diagnosing a personality disorder as a function of the individual's sociocultural environment. However, unlike the clear instructions to avoid diagnosing a personality disorder when the individual meets criteria for another mental disorder (Criterion E; p. 761), no clear instructions to avoid personality disorder diagnosis based on a comprehensive assessment of sociocultural context exist (see Criteria E, F, and G; p. 763).

The historical intra-individual deficiency model of personality disorder theory lingers today. The LPF of the AMPD illustrates how failure to sufficiently consider sociocultural context hinders generalizability of personality disorder models across populations and inadvertently propagates stigma.

\section{Sociocultural Decontextualization of the LPF Core of Personality Pathology}

“Diagnostic entities decontextualize people's problems and then recontextualize them by inventing a concept called [personality] disorder" (p. 530; Schwarz, 2018)

Personality disorders are purportedly defined by "fundamental disturbances of self and interpersonal relations" (p. 332; Bender et al., 2011) which comprise the self-interpersonal dimensions that constitute the LPF. The LPF model of personality disorder reflects intraindividual conceptualization divorced from extra-individual, structural determinants of 
functioning. For example, according to the LPF, an individual meets criteria for extreme-level intimacy impairment if they demonstrate "... profound disinterest or expectation of harm" (p. 316) in relation to interpersonal relationships (Table 1; Sharp \& Wall, 2021). Though the model itself does not wade into defining etiological underpinnings of these behavioral indicators, the ways in which personality pathology are colloquially discussed suggest an inherently biologically determined framework. The LPF is positioned as the core of personality pathology (i.e., the disease) that gives rise to observed psychosocial impairment associated with personality disorder (i.e., the disability; Sharp \& Wall, 2021).

The sociocultural context in which SGM individuals exist is one that primes individuals to expect social rejection and enact behaviors to avoid harm. Hypervigilance and sensitivity to potential rejection among SGM individuals is highlighted within theories of minority stress processes (e.g., Hatzenbuehler, 2009; Meyer, 2003; Tan et al., 2020). In his review, Feinstein (2020) notes how sensitivity to rejection can be adaptive in the face of chronic stigma from a heteronormative society. Specifically, vigilance of potential rejection helps minoritized individuals avoid unsafe situations. In addition, some literature suggests decreases in secure attachment experiences as a function of social rejection (Cook \& Calebs, 2016), with potential to impact interpersonal relationships and intimacy. How does the LPF, and the wider AMPD, contend with the fact that for SGM individuals, the sociocultural environment is one which systematically exposes them to experiencing high levels of stigma, discrimination, and victimization with impact on self- and interpersonal functioning? By divorcing itself from context, how much does the AMPD (re)stigmatize SGM individuals by potentially attributing dysfunction to an intra-individual cause (i.e., personality) rather than expectable coping mechanisms in the face of chronic stress? For instance, borderline personality disorder is 
identified as reflecting the core of personality pathology and overlaps with the LPF in several ways. We've previously suggested that sociocultural context might explain several borderline personality disorder criteria among SM populations (Rodriguez-Seijas et al., 2021a. 2021b). The sociocultural context is - at least part of - the disease process; what is conceptualized as personality pathology might be the disability.

\section{AMPD Intervention Implications and Sociocultural Context}

Failure to appropriately situate sociocultural context in assessment and diagnosis extends to intervention approaches for personality pathology. Widiger and Hines acknowledge the relative lack of evidence-based intervention approaches for maladaptive personality domains, with the exception of the neuroticism/negative affectivity domain. They direct readers to references that speculate on how extant evidence-based approaches may be adapted for different AMPD Criterion B domains. Missing from many of these approaches is explicit consideration of the ways in which sociocultural context impacts treatment conceptualization and delivery. Interventions for SGM populations highlight and embrace minority stress theory in their conceptualization and treatment approaches (e.g., Burton et al., 2019; Pachankis et al., 2019). The importance of attending to minority stress context has even been extended to dialectical behavior therapy for SGM individuals with a diagnosis of borderline personality disorder (see Pantalone et al., 2019). After summarizing myriad evidence-based approaches for psychosocial dysfunction among SM populations, Rogers and colleagues (2021) conclude that interventions share — among other things — a fundamental focus and understanding of the ways in which sociocultural context impacts the development and persistence of psychosocial dysfunction and necessarily informs treatment. Stemming from the epistemic exclusion of sociocultural context within the assessment and diagnosis of personality pathology within the AMPD, and broadly 
across personality disorder theory in general, how will the field move forward to harnessing more contextualized intervention approaches for personality disorders?

\section{Conclusion: A Call for Sociocultural Context in the AMPD and Beyond}

In discussing intersectionality theory, Cole (2009) asks scientists to consider who is included in our categories. Grzanka (2020) proposes three additional questions for the application of intersectionality theory within psychological science related to addressing systems and not solely identities; examining how social power operates within structures; and questioning the role psychologists can play in solving social problems. These questions are also important for improving and advancing personality theory related to the AMPD. Who has been excluded within the personality disorder discourse? I would venture to say that the modal study of personality disorder has failed to represent historically excluded populations like sexual, gender, and racial/ethnic minority groups. Further, I would be surprised if attention to sociocultural context, rather than inherently operating from intra-individual deficiency models, was accommodated in studies that center these underrepresented groups. How has the AMPD—and personality disorder literature more broadly — considered sociocultural context within its epistemology? How have the dominant scientific voices and their relative positions influenced the resultant personality disorder discourse? And finally, what can we do to effectively address sociocultural context within personality pathology models like the AMPD? The epistemic exclusion of sociocultural context within AMPD theory, research, and treatment is tantamount to an issue of injustice. Without contending with the socially contextualized nature of personality pathology and the impact of structural determinants of health beyond intra-individual deficiency models, the AMPD and any models of personality pathology, cannot appropriately generalize to diverse populations and risk facilitating harm to those communities. 


\section{References}

American Psychiatric Association, A. P. (2013). Diagnostic and statistical manual of mental disorders: DSM 5. American Psychiatric Association.

Bender, D. S., Morey, L. C., \& Skodol, A. E. (2011). Toward a model for assessing level of personality functioning in DSM-5, part I: A review of theory and methods. Journal of Personality Assessment, 93(4), 332-346.

Burton, C. L., Wang, K., \& Pachankis, J. E. (2019). Psychotherapy for the Spectrum of Sexual Minority Stress: Application and Technique of the ESTEEM Treatment Model. Cognitive and Behavioral Practice, 26(2), 285-299. https://doi.org/10.1016/j.cbpra.2017.05.001

Cole, E. (2009). Intersectionality and Research in Psychology. The American Psychologist, 64, 170-180. https://doi.org/10.1037/a0014564

Cook, S. H., \& Calebs, B. J. (2016). The Integrated Attachment and Sexual Minority Stress Model: Understanding the Role of Adult Attachment in the Health and Well-Being of Sexual Minority Men. Behavioral Medicine, 42(3), 164-173. https://doi.org/10.1080/08964289.2016.1165173

Crocq, M.-A. (2013). Milestones in the history of personality disorders. Dialogues in Clinical Neuroscience, 15(2), 147-153.

Feinstein, B. A. (2020). The Rejection Sensitivity Model as a Framework for Understanding Sexual Minority Mental Health. Archives of Sexual Behavior, 49(7), 2247-2258. https://doi.org/10.1007/s10508-019-1428-3

Grzanka, P. R. (2020). From Buzzword to Critical Psychology: An Invitation to Take Intersectionality Seriously. Women \& Therapy, 43(3-4), 244-261. https://doi.org/10.1080/02703149.2020.1729473 
Hatzenbuehler, M. L. (2009). How does sexual minority stigma "get under the skin"? A psychological mediation framework. Psychological Bulletin, 135(5), 707.

Meyer, I. H. (2003). Prejudice, social stress, and mental health in lesbian, gay, and bisexual populations: Conceptual issues and research evidence. Psychological Bulletin, 129(5), 674.

Pachankis, J. E., McConocha, E. M., Reynolds, J. S., Winston, R., Adeyinka, O., Harkness, A., Burton, C. L., Behari, K., Sullivan, T. J., Eldahan, A. I., Esserman, D. A., Hatzenbuehler, M. L., \& Safren, S. A. (2019). Project ESTEEM protocol: A randomized controlled trial of an LGBTQ-affirmative treatment for young adult sexual minority men's mental and sexual health. BMC Public Health, 19(1), 1086. https://doi.org/10.1186/s12889-0197346-4

pantalone, D. W., Sloan, C. A., \& Carmel, A. (2019). Dialectical Behavior Therapy for Borderline Personality Disorder and Suicidality Among Sexual and Gender Minority Individuals. In J. Pachankis \& S. A. Safren (Eds.), Handbook of Evidence-Based Mental Health Practice with Sexual and Gender Minorities (pp. 408-429). Oxford University Press.

Rodriguez-Seijas, C., Burton, C. L., \& Pachankis, J. E. (2019). Transdiagnostic Approaches to Improve Sexual Minority Individuals' Co-occurring Mental, Behavioral, and Sexual Health. In J. E. Pachankis \& S. A. Safren (Eds.), Handbook of Evidence-Based Mental Health Practice with Sexual and Gender Minorities (pp. 457-476). Oxford University Press.

Rodriguez-Seijas, C., Eaton, N. R., \& Pachankis, J. E. (2019). Prevalence of psychiatric disorders at the intersection of race and sexual orientation: Results from the National 
Epidemiologic Survey of Alcohol and Related Conditions-III. Journal of Consulting and Clinical Psychology, 87(4), 321-331. https://doi.org/10.1037/ccp0000377

Rodriguez-Seijas, C., Morgan, T. A., \& Zimmerman, M. (2021a). Is There a Bias in the Diagnosis of Borderline Personality Disorder Among Lesbian, Gay, and Bisexual Patients? Assessment, 28(3), 724-738. https://doi.org/10.1177/1073191120961833

Rodriguez-Seijas, C., Morgan, T. A., \& Zimmerman, M. (2021b). A Population-Based Examination of Criterion-Level Disparities in the Diagnosis of Borderline Personality Disorder Among Sexual Minority Adults. Assessment, 28(4), 1097-1109. https://doi.org/10.1177/1073191121991922

Rodriguez-Seijas, C., Stohl, M., Hasin, D. S., \& Eaton, N. R. (2015). Transdiagnostic factors and mediation of the relationship between perceived racial discrimination and mental disorders. JAMA Psychiatry, 72(7), 706-713.

Rogers, B. G., Harkness, A., \& Rodriguez-Seijas, C. (2021). Sexual Minority Mental Health. In Reference Module in Neuroscience and Biobehavioral Psychology. Elsevier. https://doi.org/10.1016/B978-0-12-818697-8.00146-1

Schwarz, S. (2018). Resilience in psychology: A critical analysis of the concept. Theory \& Psychology, 28(4), 528-541. https://doi.org/10.1177/0959354318783584

Sharp, C., \& Wall, K. (2021). DSM-5 Level of Personality Functioning: Refocusing Personality Disorder on What It Means to Be Human. Annual Review of Clinical Psychology, 17(1), 313-337. https://doi.org/10.1146/annurev-clinpsy-081219-105402

Tan, K. K. H., Treharne, G. J., Ellis, S. J., Schmidt, J. M., \& Veale, J. F. (2020). Gender Minority Stress: A Critical Review. Journal of Homosexuality, 67(10), 1471-1489. https://doi.org/10.1080/00918369.2019.1591789 